Research Article

\title{
Routing Optimisation of Urban Medical Waste Recycling Network considering Differentiated Collection Strategy and Time Windows
}

\author{
Jiajing Gao, Haolin Li, Jingwen Wu $\left(\mathbb{D}\right.$, Junyan Lyu, Zheyi Tan $\mathbb{D}^{D}$, and Zhufan Jin \\ School of Management, Shanghai University, Shanghai, China \\ Correspondence should be addressed to Jingwen Wu; jingwen_wu@shu.edu.cn
}

Received 11 January 2021; Revised 23 February 2021; Accepted 6 March 2021; Published 18 March 2021

Academic Editor: Tingsong Wang

Copyright (c) 2021 Jiajing Gao et al. This is an open access article distributed under the Creative Commons Attribution License, which permits unrestricted use, distribution, and reproduction in any medium, provided the original work is properly cited.

\begin{abstract}
The increasing gap between medical waste production and disposal stresses the urgency of further development of urban medical waste recycling. This paper investigates an integrated optimisation problem in urban medical waste recycling network. It combines the vehicle routing problem of medical facilities with different requirements and the collection problem of clinics' medical waste to the affiliated hospital. To solve this problem, a compact mixed-integer linear programming model is proposed, which takes account of the differentiated collection strategy and time windows. Since the medical waste recycling operates according to a two-day pattern, the periodic collection plan is also embedded in the model. Moreover, we develop a particle swarm optimisation (PSO) solution approach for problem-solving. Numerical experiments are also conducted to access the solution efficiency of the proposed algorithm, which can obtain a good solution in solving large-scale problem instances within a reasonable computation time. Based on the results, some managerial implications can be recommended for the third-party recycling company.
\end{abstract}

\section{Introduction}

Medical waste contains potentially dangerous microorganisms produced by medical activities. If medical waste is not properly recycled and disposed, it may pollute people's living environment, spread diseases, and sometimes pose threats to public health [1]. It is particularly important to establish a safe, sustainable, and green medical waste recycling system to protect human health, maintain ecological safety, and promote sustainable development. According to the data of the National Bureau of Statistics shown in Figure 1, the production of medical waste in 2008 was about 1.222 million tons, and, in 2017, the production of medical waste was 2.235 million tons, $183 \%$ of that in 2008 . However, the recycling rate of medical waste is still in a low level, where about twothirds of the medical waste are not properly disposed. The gap between medical waste production and disposal is still significant. The main problem of medical waste collection is the lack of proper facilities for waste collection, safe storage, and transportation, which stresses the urgency of further development of medical waste recycling [2]

The recycling of medical waste can be divided into two stages, namely, collection stage and recycling and disposing stage. In the collection stage, medical waste is collected from medical facilities. In the recycling and disposing stage, waste is recycled and disposed in the recycling centre. Both stages stress the safe in both transportation and disposal, which will be supervised by the government and the public. The collection stage includes three key steps: classification, temporary storage, and transport. Among those steps, the transportation step consumes much labour and resources. Effective planning is required in the transportation step to improve efficiency and cut down the operational cost for the medical waste collection operators. Vehicle routing problem (VRP) is a well-known optimisation problem in transportation section. In medical waste recycling network, the operator needs to determine the route of collection vehicle. Before applying the vehicle routing problem into the medical 


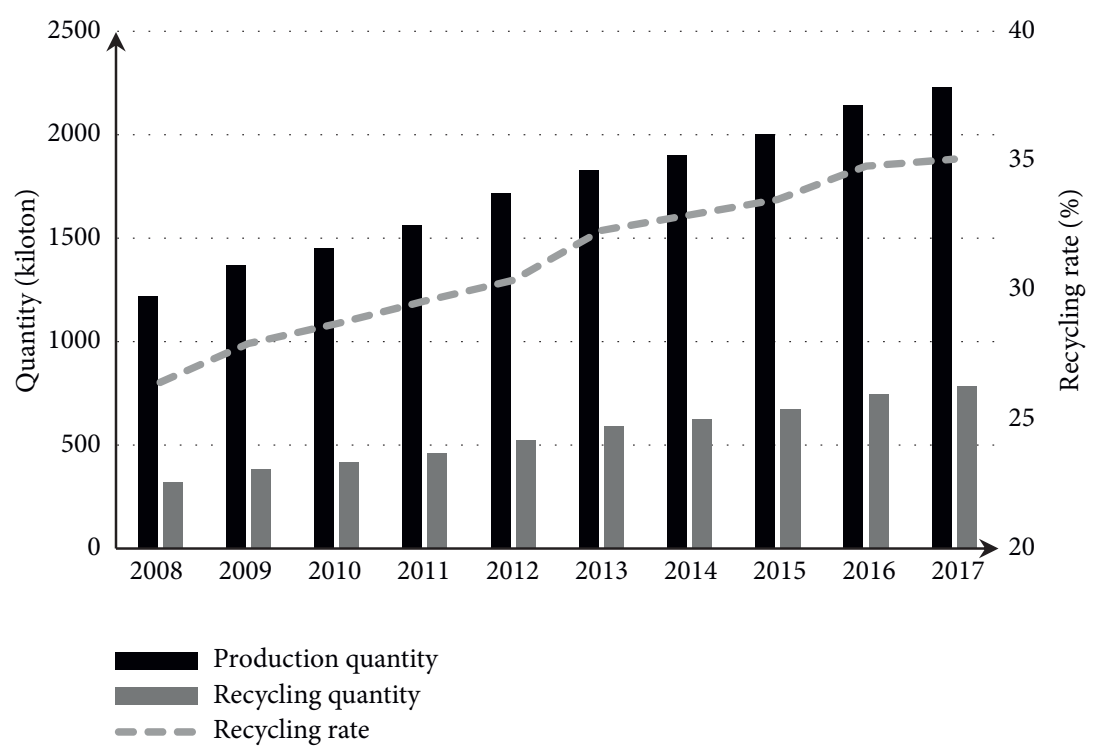

FIGURE 1: Statistics data of medical waste production.

waste recycling network, we should pay attention to the difference of medical waste collection compared to general waste collection, that is, the potential risk in transportation and storage process of medical waste. To reduce the potential storage risk of storage, a higher service frequency is required in medical waste network. The structure of medical waste collection network may also have some special feature. Based on the differences, some new challenges, such as the periodicity of waste collection, regulations for the storage and transportation of medical waste, and the different waste quantity produced by different medical facilities, need to be tackled. These challenges imply that the medical waste recycler should provide the differentiated waste collection strategy, which can be achieved by different service frequencies. In this paper, we focus on the routing optimisation of a typical urban medical waste recycling network in China and build up a mixed-integer linear programming model to solve the periodic vehicle routing problem with time windows (PVRPTW) with the objective of minimising the total travelling distance. Based on the periodicity of waste collection and the different requirements of medical facilities, we propose a differentiated recycling strategy to collect medical waste. To enhance the computing efficiency, PSO algorithm is applied for problem-solving. Numerical experiments are used to verify the model and the effectiveness of the proposed algorithm, and sensitivity analysis is conducted to explain some related problems.

The rest of this paper is organised as follows: Section 2 provides an exhaustive literature review of related research. Section 3 expounds the research problems of this paper and describes the special features of the routing problem in medical waste network. Section 4 elaborates the mathematical model. Section 5 proposes PSO algorithm for problem-solving. Section 6 conducts a numerical experiment to verify the model and algorithm and puts forward some management insights by sensitivity analysis. Section 7 presents the conclusion.

\section{Literature Review}

Medical waste collection and recycling problems can be defined as a PVRPTW, which determines minimum distance routes in a reverse logistics network. Among the research problems in reverse logistics, VRP is one of the most important and most widely studied combinatorial optimisation problems because of its complexity and importance in cost minimisation in transportation networks [3]. The spread of COVID-19 puts the whole world into danger and panic, and, of course, the amount of medical waste from COVID-19 encounters explosive growth, which places pressure on the waste disposing systems and poses a great threat of virus infection to the public. When focusing on the research of medical waste recycling, we find that COVID-19 and other public health events have motivated scholars to conduct research about medical waste recycling. Among the previous researches, waste collection problems in previous works are usually modelled as inventory-routing problem, locationrouting problem, and supply chain network construction problem. Cost and social objectives like public risk are taken as the objective of optimisation problems.

Inventory-routing problem can be seen as a special form of VRP, where medical waste in medical facilities is waiting to be collected. Nolz et al. [4] investigate the collection of medical waste stored at pharmacies. Stochastic aspects are considered in the inventory-routing problem, and the social objective is applied as the objective. A sampling method and an approach based on an adaptive large neighbourhood search algorithm are developed to solve the proposed problem.

Location-routing problem is another hotspot of medical waste recycling network. Mantzaras and Voudrias [5] develop an optimisation model to minimise the cost of infectious waste management. Location and capacity of treatment plants and transfer stations as well as transport paths of vehicles are determined in this model. 
Tirkolaee et al. [6] investigate a sustainable multitrip location-routing problem with time windows for medical waste management. Travelling time, total violation of time windows, service priorities, total infection, and environmental risk are minimised in their model. Fuzzy chance-constrained programming approach is applied to address uncertainty. The model is then tested by a case study in Sari city of Iran.

Some scholars investigate medical waste recycling networks as a supply chain network design and construction problems. Yu et al. [7] propose a multiobjective multiperiod mixed-integer programming model for the epidemic logistics network of medical waste to determine facility locations and transportation strategies. Risk in transportation and treatment and the cost of system establishment are selected as the objectives. Several general policies are obtained by experiments and analysis. Kargar et al. [8] propose a multi-item and multiperiod linear programming model for medical waste reverse supply chain with objectives relevant to total costs, treatment technology selection, and medical waste stored. A robust possibilistic programming approach and a fuzzy goal programming method are employed in modelling.

VRP is a well-known NP-hard problem. To obtain a solution in a reasonable time, researchers try to use heuristic algorithms to solve the models and obtain near-optimal solutions efficiently. Global search algorithms like genetic algorithm, PSO, and simulated annealing are applied because of the strong capability of finding the global optimum [9-11]. For local search heuristics, some metaheuristics are applied in problem-solving to improve search efficiency and avoid trapping in local optimum, like Squeaky Wheel Optimisation [12,13] and Critical-Shaking Neighbourhood Search $[14,15]$.

From the literatures reviewed above, we can find that medical waste collection and disposing is an emerging research field in reverse logistics. Although many works have been done about VRP considering time windows and vehicle capacity in the field of waste collection, to the best of our knowledge, there are few works that investigate the differentiated collection strategy for medical facilities. The main contribution of our research can be categorized into three aspects. First, we investigate the PVRPTW of medical waste recycling network considering the differentiated collection strategy based on different grades of medical facilities, in which different collection frequency and collection from clinics to grade 1 hospital are applied. A mixed-integer linear programming model is formulated to minimise the total travelling distance. Second, we propose a PSO algorithm for problem-solving, which can be a tool for solving real-scale problems. Third, some managerial insights about vehicle capacity are concluded according to numerical experiments and sensitivity analysis, which provide a broad view of network operation.

\section{Problem Background}

This study attempts to investigate the PVRPTW of an urban medical waste recycling network based on a typical urban medical system in China. The urban medical waste recycling network works to recycle waste produced in the daily operations of medical facilities. There are several medical facilities and a recycling centre in the network. The medical facilities are the producers of medical waste, including hospitals in different grades, clinics, and other medical waste producers. Medical waste produced by medical facilities is recycled or disposed by the recycling centre. Once the medical waste is produced, it is stored in temporary storage areas within or adjacent to medical facilities and waits to be collected. Vehicles for medical waste collection start from the recycling centre, go to assigned hospitals to collect waste along the assigned routes, and go back to the recycling centre when all wastes in the assigned hospitals are collected. According to regulations, the medical waste can only be stored in the temporary storage areas for a maximum of 48 hours to minimise the risk of infections [16].

In general, medical facilities have different functions and sizes, and the amount of medical waste produced by different facilities varies. For economic reasons, different waste quantity among medical facilities requires different frequency of waste collection; that is to say, the medical facilities that produce more waste require higher frequency of waste collection. Based on the hospital classification system and the actual operation of the medical system, we classify the medical facilities into the following 4 grades in our research and set the corresponding collection strategies to different grade of medical facilities.

(1) Grade 2 and Above Hospitals. These hospitals are the core of the medical system, which will treat more patients and produce more medical waste in daily operation. These hospitals all have temporary storage areas for waste, so the waste can be stored in the temporarily storage areas within the hospitals, and the vehicles need to visit these hospitals to collect waste. To deal with the large amount of waste, we set a higher waste collection frequency for grade 2 and above hospitals as once a day.

(2) Grade 1 Hospitals. These hospitals are usually community hospitals and health centres and are distributed more densely in the city. The wastes produced by grade 1 hospitals are stored in their temporary storage area and collected by the vehicles every two days. Grade 1 hospitals are also used as collection points for those clinics without the temporary storage areas because of the denser distribution.

(3) Clinics. The clinics are more common in the city and appear as supplements of the medical system. Clinics are usually smaller and generate less medical waste, so there are no medical waste temporary storage areas in clinics. Once waste is produced by clinics, it is sent to temporary storage areas in nearby grade 1 hospitals and waits to be collected. This requires an affiliated relationship between clinics and grade 1 hospitals. The clinics can affiliate a grade 1 hospital only when the clinic locates in the service radius of the hospital. Since the distances between clinics and 
their affiliated hospitals are short, the cost incurred by waste collection of clinics is ignored in our research.

(4) Other Waste Producers. Some medical waste is produced by some facilities other than hospitals and clinics, including scientific institutions and laboratories, blood banks, and pharmaceutical manufacturers. These facilities have temporary storage areas for waste, and the waste is collected every two days.

Based on the settings above, a two-day period can be set up for waste collection. Each period contains two days, namely, odd day and even day. Waste collection tasks are generated among hospitals according to the waste collection frequency and waste amount. For every waste collection period, grade 2 and above hospitals will generate two waste collection tasks for the waste produced by themselves: one task involves the collection of waste produced on the odd day, and the other task involves the collection of waste produced on the even day. Grade 1 hospitals will generate one collection task in a period, which contains the waste produced by themselves and the affiliated clinics. The task of grade 1 hospitals can be collected on either odd day or even day. The task of other waste producers contains the waste produced in two days and will be collected once in a period. The waste collection and recycling activities follow certain working hours; that is to say, a time window is added to the route of waste collection.

Based on the above process, the medical waste transport network is shown in Figure 2. The above problem setting can be abstracted as a PVRPTW, in which the recycling centre serves as the depot of the network, and medical facilities play the role of the nodes where demands are located. All collection routes are visited on either odd day or even day and start from and end at the recycling centre. Every collection task should be served in one of the two days and assigned to a specific route. The objective of this problem is to minimise the total transportation distance. The decision problem contains the collection routes of vehicles along with the date and time of tasks being served. For the waste collection of clinics, the affiliated relationship also needs to be determined.

\section{Mathematical Model}

4.1. Notations. The notations used to formulate the model are introduced below.

Indices and Sets

$w, w^{\prime}:$ Index of task, which means the medical waste to be collected in a hospital

$W$ : Set of all tasks

$W^{0}$ : A subset of task set $W, W^{0} \subseteq W, 2 \leq\left|W^{0}\right| \leq|W|$

$i$ : Index of hospitals

I: Set of all hospitals

$I_{g}$ : Set of grade $g$ hospitals; in detail, $I_{0}$ represents other medical facilities, $I_{1}$ represents set of grade 1 hospitals, and $I_{2}$ represents grade 2 and above hospitals

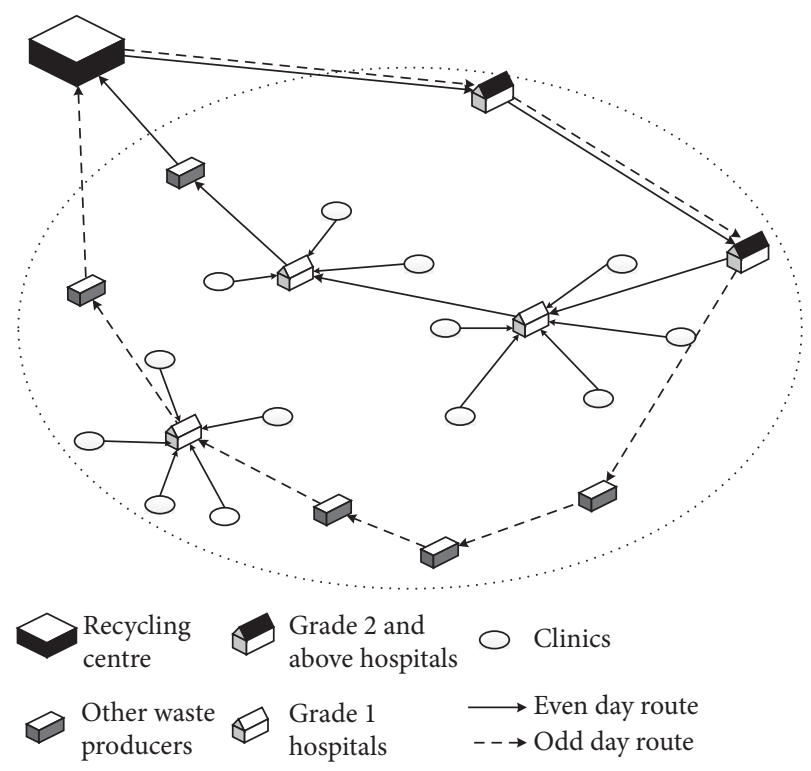

FIGURE 2: Hierarchy diagram of medical waste recovery network.

\section{$j$ : Index of clinics \\ $J$ : Set of all clinics \\ $t$ : Index of date}

$T$ : Set of dates in a transport cycle. $T=\{1,2\}$, where 1 represents odd day, and 2 represents even day

$r$ : Index of vehicles

$R$ : Set of all vehicles

$R_{t}$ : Set of vehicles working on date $t ; R_{1}$ represents vehicles working on odd days, and $R_{2}$ represents vehicles working on even days.

\section{Parameters}

$e(r), e^{\prime}(r)$ : Recycling centre, the start point, and end point for collection route

$h_{w}$ : Corresponding hospital of task $w$

$g_{i}$ : Grade of hospital $i$, where 0 represents other waste producers, 1 represents grade 1 hospitals, and 2 represents grade 2 and above hospitals

$d_{w, w^{\prime}}$ : Travelling distance between tasks $w$ and $w^{\prime}$

$t_{w, w^{\prime}}$ : Travelling time between tasks $w$ and $w^{\prime}$

$p_{j, i}$ : Set to 1 if the clinic $j$ locates within the service radius of grade 1 hospital $i$ and 0 otherwise

$t_{w}$ : Task $w$ 's processing time

$\left[a^{f}, b^{f}\right]$ : The availability service time of waste collection for all tasks

$q_{w}^{1}$ : The amount of medical waste produced by the hospital corresponding to the task $w$

$q_{j}^{2}$ : The amount of medical waste in the clinic $j$

$Q_{i}^{1}$ : Medical waste capacity of grade 1 hospital $i, i \in I_{1}$

$Q_{r}^{2}$ : Maximum capacity of vehicle $r, r \in R$

$M$ : A sufficiently large positive number

$H: 24$ hours. 


\section{Decision Variables}

$\mu_{j, i}$ : Binary variable, set to 1 if the waste of clinic $j$ is collected to the hospital $i$ and 0 otherwise

$\pi_{w, w^{\prime}, r}$ : Binary variable, set to 1 if the vehicle $r$ serves the task $w^{\prime}$ immediately after serving the task $w$ and 0 otherwise

$\varphi_{w, t}$ : Binary variable, set to 1 if the task $w$ is served in date $t$ and 0 otherwise

$\sigma_{w, r}$ : Binary variable, set to 1 if the task $w$ is served by the vehicle $r$ and 0 otherwise $\rho_{w, r}:$ Float variable, the start time of the task $w$ being served by vehicle $r$

$\tau_{w}$ : Float variable, the total medical waste of the task $w$ $\varepsilon_{w, r}$ : Float variable, the amount of medical waste in the task $w$ transported by the vehicle $r$.

Based on the notations above, to simplify the expression, we define the set $W_{i}$ to express task set of the hospital $i$, where $W_{i}=\left\{w \mid w \in W, h_{w}=i\right\}$.

\subsection{Mathematical Model Construction.}

$$
\begin{aligned}
& \text { Minimize } \sum_{r \in R} \sum_{w \in W \cup\{e(r)\}} \sum_{w^{\prime} \in W \cup\left\{e^{\prime}(r)\right\}} d_{w, w^{\prime}} \pi_{w, w^{\prime}, r}, \\
& \text { s.t. } \quad \sum_{i \in I_{1}} \mu_{j, i}=1, \forall j \in J \text {, } \\
& \mu_{j, i} \leq p_{j, i}, \quad \forall i \in I_{1}, j \in J, \\
& \sum_{r \in R} \sigma_{w, r}=1, \quad \forall w \in W \\
& \sigma_{w, r} \leq \varphi_{w, t}, \quad \forall w \in W, t \in T, r \in R_{t}, \\
& \sum_{w \in W \cup\{e(r)\}} \pi_{w, w^{\prime}, r}=\sum_{w \in W \cup e^{\prime}(r)} \pi_{w_{1}, w, r}=\sigma_{w_{1}, r}, \quad \forall w^{\prime} \in W, r \in R, \\
& \sum_{w \in W \cup\left\{e^{\prime}(r)\right\}} \pi_{e(r), w, r}=\sum_{w \in W \cup\{e(r)\}} \pi_{w, e_{1}(r), r}=1, \quad \forall r \in R, \\
& \sum_{w \in W^{0}} \sum_{w^{\prime} \in W^{0}} \pi_{w, w^{\prime}, r} \leq\left|W^{0}\right|-1 W^{0} \subseteq W, \quad 2 \leq\left|W^{0}\right| \leq|W|, \forall r \in R, \\
& \tau_{w}=q_{w}^{1}+\sum_{j \in J} q_{j}^{2} \mu_{j, i}, \quad \forall w \in W, i \in I_{1} \\
& \tau_{w}=q_{w}^{1}, \quad \forall w \in W, i \in I_{0} \cup I_{2}, \\
& \tau_{w} \leq Q_{i}^{1}, \quad \forall w \in W, i \in I_{1}, \\
& \varepsilon_{w, r} \leq M \sigma_{w, r}, \quad \forall w \in W, r \in R, \\
& \varepsilon_{w, r} \leq \tau_{w}, \quad \forall w \in W, r \in R, \\
& \varepsilon_{w, r} \geq \tau_{w}-M\left(1-\sigma_{w, r}\right), \quad \forall w \in W, r \in R, \\
& \sum_{w \in W} \varepsilon_{w, r} \leq Q_{r}^{2}, \quad \forall r \in R, \\
& \varphi_{w, 1}+\varphi_{w, 2}=1, \quad \forall w \in W
\end{aligned}
$$




$$
\begin{gathered}
\sum_{w \in W_{i}} \varphi_{w, t} \leq 1, \quad \forall t \in T, i \in I, \\
\sum_{w \in W_{i}} \varphi_{w, 1}+\sum_{w \in W_{i}} \varphi_{w, 2}=2, \quad \forall i \in I_{2}, \\
\sum_{w \in W_{i}} \varphi_{w, 1}+\sum_{w \in W_{i}} \varphi_{w, 2} \geq 1, \quad \forall i \in I_{0} \cup I_{1}, \\
\rho_{w, r} \geq a^{f}+t_{w}+H \varphi_{w, 2}+M\left(\sigma_{w, r}-1\right), \quad \forall w \in W, r \in R, \\
\rho_{w, r} \leq b^{f}+H \varphi_{w, 2}-M\left(\sigma_{w, r}-1\right), \quad \forall w \in W, r \in R, \\
\rho_{w, r} \leq M \sigma_{w, r}, \quad \forall w \in W, r \in R, \\
\rho_{w^{\prime}, r} \geq \rho_{w, r}+t_{w^{\prime}}+t_{w, w^{\prime}}-M\left(1-\pi_{w, w^{\prime}, r}\right), \quad \forall w \in W \cup\{e(r)\}, w^{\prime} \in W \cup\left\{e^{\prime}(r)\right\}, r \in R, \\
\mu_{j, i}, \pi_{w, w_{l}, r}, \sigma_{w, r} \in\{0,1\}, \quad \forall w \in W \cup\{e(r)\}, w w^{\prime} W \cup\left\{e^{\prime}(r)\right\}, r \in R, i \in I, j \in J, t \in T, \\
\rho_{w, r}, \tau_{w}, \varepsilon_{w, r} \geq 0, \quad \forall w \in W \cup\left\{e(r), e^{\prime}(r)\right\}, r \in R .
\end{gathered}
$$

The objective function of this model is to minimise the total transportation distance, which is shown in constraint (1). Constraints (2) determine the affiliation between the clinic and the level 1 hospital, where a clinic must be affiliated to only one grade 1 hospital. Constraints (3) indicate that a clinic can affiliate with a grade 1 hospital only when the clinic is in the coverage area of the hospital. Constraints (4) make sure that all tasks will be served by a vehicle. Constraints (5) state that a vehicle can serve a task only when the vehicle works on the date that the task is assigned. Constraints (6) and (7) ensure the routes' consecutiveness of all vehicles, and constraints (8) prevent the shipping routes of vehicles forming loops. Constraints (9) ensure that, for all tasks of grade 1 hospitals, the total amount of waste to be transported in the corresponding task equals the sum of the wastes produced by itself and collected from the affiliated clinics. Constraints (10) state that, for all tasks of grade 0 and 2 hospitals, the total amount of medical waste to be transported in the corresponding task equals the amount of waste produced by itself. Constraints (11) make sure that the total amount of medical waste in each task cannot exceed the maximum capacity of the corresponding hospital. Constraints (12), (13), and (14) denote the shipping amount of task $w$ by vehicle $r$. If task $w$ is assigned to be shipped by vehicle $r$, the shipping amount should be equal to the total waste amount of the task. If the task $w$ will not be shipped by vehicle $r$, the shipping amount must be zero. Constraints (15) imply that the total amount of medical waste transported by a vehicle should not exceed its maximum capacity. Constraints (16) and (17) ensure that a task should be assigned to only one date. Constraints (18) ensure that a grade 2 and above hospital must have two transportation tasks within a transport cycle. Constraints (19) ensure that level 0 and level 1 hospitals have one transportation task within a transport cycle. Constraints (20) and (21) set the working time limit on vehicles. Constraints (22) denote that the start time of the task $w$ served by vehicle $r$ can exist only if the task $w$ is assigned to be served by vehicle $r$. Constraints (23) indicate the relationship of start time for adjacent tasks. Constraints (24) and (25) define all decision variables.

\section{Solution Method}

5.1. PSO Solution Method. For small-scale instances, the proposed model can be solved directly by CPLEX and some other commercial solvers. However, the commercial solver cannot solve large-scale problem instances within a reasonable time. Therefore, we design a PSO solution approach to solve the problem. PSO is widely used for solving continuous nonlinear optimisation problems because of its simple concept, easy implementation, and quick convergence [17]. The PSO algorithm is successfully applied by numerous researchers to solve VRP $[18,19]$. For the problem in other logistics sections, PSO can also achieve outstanding performance [20].

For the PSO method, we employ dimensional reduction for the particles to improve the solving efficiency. The first group of particles is defined by variables $\mu_{j, i}$ with the notation $\mu_{m j}^{n}$. For a particle $m$ in iteration $n, \mu_{m j}^{n}$ denotes the hospital $i$ where the medical waste of the clinic $j$ is collected. Similarly, the second group of particles is defined by variables $\varphi_{w, t}$ with the notation $\varphi_{m w}^{n}$. For a particle $m$ iteration $n$, $\varphi_{m w}^{n}$ denotes the date $t$ where the task $w$ is served. Variables $\pi_{w, w^{\prime}, r}$ and $\sigma_{w, r}$ can be redefined by $\beta_{m r}^{n}$ and $\pi_{m r z}$, where $\beta_{m r}^{n}$ represents the number of tasks which is assigned to the vehicle $r$ and $\pi_{m r z}$ represents the task $w$ served by vehicle $r$ in sequence $z$. To determine the sequence of tasks and connect the two particles $\beta_{m r}^{n}$ and $\pi_{m r z}$, we introduce the priority 
attribute $\chi_{w} \cdot \chi_{w}$ represents the priority of task $w$. The value of $\chi_{w}$ is randomly generated within an interval, which is used to determine the sequence $z$ in particle $\pi_{m r z}$. A random sequence can be indicated from the set containing all $\chi_{w}$; that is, the task with smaller value of $\chi_{w}$ will be processed earlier. For example, there are 3 tasks named $w_{1}, w_{2}$, and $w_{3}$ waiting to be served by the vehicle $r$, or we can express this by $\beta_{m r}^{n}=3$. To determine the sequence of the three tasks, $\chi_{w_{1}}=53, \chi_{w_{2}}=72$, and $\chi_{w_{3}}=20$ are randomly generated within the interval $[0,100]$. According to the value of $\chi_{w}$, the sequence of the vehicle serving those tasks is $w_{3} \longrightarrow w_{1} \longrightarrow w_{2}$; that is, $\pi_{m r 1}=w_{3}, \pi_{m r 2}=w_{1}$, and $\pi_{m r 3}=w_{2}$. The updating formulas of velocity and position for both groups of particles are presented as follows:

$$
\begin{aligned}
\mu V_{m j}^{n+1} & =\mu V_{m j}^{n}+c_{1} r_{1}\left(\mu p \operatorname{Best}_{m j}^{n}-\mu_{m j}^{n}\right)+c_{2} r_{2}\left(\mu g \text { Best }_{j}^{n}-\mu_{m j}^{n}\right), \\
\mu_{m j}^{n+1} & =\mu_{m j}^{n}+\mu V_{m j}^{n+1}, \\
\varphi V_{m w}^{n+1} & =\varphi V_{m w}^{n}+c_{1} r_{1}\left(\varphi p \operatorname{Best}_{m w}^{n}-\varphi_{m w}^{n}\right)+c_{2} r_{2}\left(\varphi \text { Best }_{w}^{n}-\varphi_{m w}^{n}\right), \\
\varphi_{m w}^{n+1} & =\varphi_{m w}^{n}+\varphi V_{m w}^{n+1}, \\
\beta V_{m r}^{n+1} & =\beta V_{m r}^{n}+c_{1} r_{1}\left(\beta p \text { Best }_{m r}^{n}-\beta_{m r}^{n}\right)+c_{2} r_{2}\left(\beta g \text { Best }_{r}^{n}-\beta_{m r}^{n}\right), \\
\beta_{m r}^{n+1} & =\beta_{m r}^{n}+\beta V_{m r}^{n+1}, \\
\chi V_{m w}^{n+1} & =\chi V_{m w}^{n}+c_{1} r_{1}\left(\chi p \text { Best }_{m w}^{n}-\chi_{m w}^{n}\right)+c_{2} r_{2}\left(\chi g \text { Best }_{w}^{n}-\chi_{m w}^{n}\right), \\
\chi_{m w}^{n+1} & =\chi_{m w}^{n}+\chi V_{m w}^{n+1} .
\end{aligned}
$$

In formulas 26 and $27, \mu V_{m j}^{n+1}$ and $\mu V_{m j}^{n}$ represent the current velocity and the previous velocity of particle $\mu_{m j}^{n}$ on dimension $m$, respectively. For dimension $m, \mu p$ Best $_{m j}^{n}$ denotes the best position of particle $\mu_{m j}^{n}$ on up to iteration $n$, and $\mu g$ Best $_{j}^{n}$ denotes the best position of the whole swarm until iteration $n ; \mu_{m j}^{n+1}$ and $\mu_{m j}^{n}$ denote the current and previous position of the particle. In formulas 28 and 29, $\varphi V_{m w}^{n+1}$ and $\varphi V_{m w}^{n}$ represent the current velocity and the previous velocity of particle $\varphi_{m w}^{n}$ on dimension $m$, respectively. For dimension $m, \varphi p$ Best $_{m w}^{n}$ denotes the best position of particle $\varphi_{m w}^{n}$ on up to iteration $n$, and $\varphi g$ Best $_{w}^{n}$ denotes the best position of the whole swarm until iteration $n ; \varphi_{m w}^{n+1}$ and $\varphi_{m w}^{n}$ denote the current and previous position of the particle. In formulas 30 and $31, \beta V_{m r}^{n+1}$ and $\beta V_{m r}^{n}$ represent the current velocity and the previous velocity of particle $\beta_{m r}^{n}$ on dimension $m$, respectively. For dimension $m, \beta p$ Best $_{m r}^{n}$ denotes the best position of particle $\beta_{m r}^{n}$ on up to iteration $n$, and $\beta g$ Best $_{r}^{n}$ denotes the best position of the whole swarm until iteration $n ; \beta_{m r}^{n+1}$ and $\beta_{m r}^{n}$ denote the current and previous position of the particle.

In formulas 32 and $33, \chi V_{m w}^{n+1}$ and $\chi V_{m w}^{n}$ represent the current velocity and the previous velocity of particle $\chi_{m w}^{n}$ on dimension $m$, respectively. For dimension $m, \chi p$ Best $_{m w}^{n}$ denotes the best position of particle $\varphi_{m w}^{n}$ on up to iteration $n$, and $\chi g$ Best $_{w}^{n}$ denotes the best position of the whole swarm until iteration $n ; \chi_{m w}^{n+1}$ and $\chi_{m w}^{n}$ denote the current and previous position of the particle. For both groups of particles, $c_{1}$ and $c_{2}$ are acceleration weights; $r_{1}$ and $r_{2}$ are two random numbers generated within the interval $[0,1]$.
5.2. Main Framework of the PSO Procedure. Based on the above components, the PSO procedure for the proposed problem is listed in Table 1.

\section{Numerical Experiment}

We conduct extensive numerical experiments to access the solution efficiency of the proposed algorithm. All experiments are performed on a computer with Intel Xeon E52680 v4 CPU @2.40 GHz and 256 GB RAM. The proposed model and algorithm are implemented in C\# (VS2019) concert technology with the solver IBM ILOG CPLEX 12.5.1.

6.1. Generation of the Test Instances. In the computational experiments, we test nine instances with different scales. The parameter settings for the instance groups (ISGs) are shown in Table 2. The planning horizon considered is two days. Each task is served from $0 \mathrm{am}$ to $10 \mathrm{am}$. The service radius of grade 1 hospital is 500 metres. Other input parameters for the experiments are uniformly distributed in the ranges given in Table 3.

For the PSO algorithm, the learning factors $r_{1}$ and $r_{2}$ are randomly generated within the interval $[0,1]$. Based on the results of the test runs, the acceleration weights $c_{1}$ and $c_{2}$ are both set to 0.638 .

6.2. Performance of the Proposed Solution Method. To validate the quality and efficiency of the proposed PSO algorithm, we first conduct the experiments on small-scale 
TABLE 1: PSO algorithm procedure.

Parameters: $\mathbf{n}, \mathbf{N},\left|\mathbf{I}_{0}\right|,\left|\mathbf{I}_{1}\right|,\left|\mathbf{I}_{2}\right|,|\mathbf{J}|,|\mathbf{W}|,\left|\mathbf{R}_{1}\right|,\left|\mathbf{R}_{2}\right|,|\mathbf{T}|, \mathbf{c}_{1}, \mathbf{c}_{2}, \mathbf{r}_{1}, \mathbf{r}_{2} / / n$ represents the current iteration number, $N$ represents the maximum iteration number.

Objective: $\sum_{r \in R_{1} \cup R_{2}} \sum_{w \in W \cup\{e(r)\}} \sum_{w^{\prime} \in W \cup e^{\prime}(r)} d_{w, w^{\prime}} \pi_{w, w_{l}, r}$

1 Define $\mu_{m j}^{n}, \varphi_{m w}^{n}, \beta_{m r}^{n}, \chi_{m w}^{n}, \operatorname{num}_{t_{1}}$, num $_{t}, \pi_{m r z}, \mu V_{m j}^{n}, \varphi V_{m w}^{n}, \beta V_{m r}^{n}, \chi V_{m w}^{n}, \mu p$ Best $_{m j}^{n}, \varphi p$ Best $_{m w}^{n}, \beta p$ Best $_{m r}^{n}, \chi p$ Best $_{m w}^{n}, \mu g$ Best $_{j}^{n}, \varphi g$ Best $_{w}^{n}$, $\beta g$ Best $_{r}^{n}, \chi g$ Best $_{w}^{n}$, Fitness ${ }_{m}^{n}, P$ Best $_{m}^{n}, G$ Best $^{n}$

2 For $\mathbf{m} \in \mathbf{M} / / M$ is the set of particles

3 For $\mathbf{j} \in \mathbf{J}$

4 Set $\mu_{m j}^{n}$ to a random number $i \in I_{1}, p_{j, i}=1$

5 End for

6 For $w \in W$

7 Set $\varphi_{m w}^{n}$ to a random number of intervals $[0, T]$

8 End for

9 Calculate the total number of tasks num $t_{1}$ and num $t_{2}$ in different date

10 For $\mathbf{r} \in \mathbf{R}$

11 Set $\beta_{m r}^{n}$ to a random number of intervals $\left[0\right.$, num $\left._{t_{1}}\right]$ or $\left[0\right.$, num $\left._{t_{2}}\right]$

End for

12 For $\mathbf{r} \in \mathbf{R}, \mathrm{z} \in \beta_{\mathrm{mr}}^{\mathrm{n}}$

13 Set $\pi_{m r z}$ to a random number of intervals $[0, W]$

14 End for

15 For $\mathbf{w} \in \mathbf{W}$

16 Set $\chi_{m w}^{n}$ to a random number of intervals $[0,100]$

17 End for

18 Reorder $\pi_{m r z}$ based on $\chi_{m w}^{n}$

19 If $\rho_{w, r} \in\left[a^{f}, b^{f}\right], \tau_{w} \leq Q_{i}^{1}, \varepsilon_{w, r} \leq Q_{r}^{2}$, then calculate the fitness value; otherwise calculate the fitness value with penalty cost

20 Update $P$ Best $_{m}^{n}, G$ Best $^{n}$

21 End for

22 While $(\mathbf{n}<\mathbf{N})$ do

23 For $\mathbf{m} \in \mathbf{M}$

24 Update the velocity and position of $\mu_{m j}^{n}, \varphi_{m w}^{n}, \beta_{m r}^{n}, \chi_{m w}^{n}$

25 Make $\mu_{m j}^{n}, \varphi_{m w}^{n}, \beta_{m r}^{n}, \chi_{m w}^{n}$ feasible, and reorder $\pi_{m r z}$ based on $\chi_{m w}^{n}$

26 Update $P$ Best $_{m}^{n}, G$ Best $^{n}$

27 End for

$28 \mathbf{n}=\mathbf{n}+1$

29 End while

30 Return $G$ Best $^{n}$

TABle 2: Scale of instance groups in experiments.

\begin{tabular}{lccccc}
\hline Group ID & $\begin{array}{c}\text { No. of other medical } \\
\text { facilities }\left(\left|I_{0}\right|\right)\end{array}$ & $\begin{array}{c}\text { No. of grade 1 } \\
\text { hospitals }\left(\left|I_{1}\right|\right)\end{array}$ & $\begin{array}{c}\text { No. of grade } 2 \\
\text { hospitals }\left(\left|I_{2}\right|\right)\end{array}$ & No. of tasks $(|W|)$ & $\begin{array}{c}\text { No. of } \\
\text { clinics }(|J|)\end{array}$ \\
\hline vehicles $\left(\left|R_{t}\right|\right)$
\end{tabular}

instances by comparing the results using the CPLEX solver. From Table 4, we can observe that the PSO algorithm and the CPLEX solver obtain the same objective results. However, the optimal solutions calculated by CPLEX are achievable within 2 hours only for ISG1 and ISG2, which means the CPLEX can only solve some small-scale instances. The PSO algorithm performs more efficiently than CPLEX in terms of computation time, where the average computing time for PSO is only 15.61 seconds.
For the medium-scale instances in Table 5, we can conclude that, for medium-scale instances, the solutions obtained by PSO are at least as good as the feasible solution obtained by CPLEX in 7200 seconds, while the computation process of PSO algorithm is much faster than CPLEX.

When facing large-scale instances in our experiments, a set of assignment rules are conducted for comparison. The main procedure of the rules is listed as follows. 
TABLE 3: Parameters setting for the experiments.

\begin{tabular}{lc}
\hline Parameter & Value \\
\hline$d_{w, w^{\prime}}$ & {$[1-3] \mathrm{km}$} \\
$q_{j}^{2}$ & {$[3-6] \mathrm{kg}$} \\
$t_{w \in W_{0}}$ & {$[0.05-0.10] \mathrm{h}$} \\
$t_{w \in W_{1}}$ & {$[0.10-0.17] \mathrm{h}$} \\
$t_{w \in W_{2}}$ & {$[0.17-0.27] \mathrm{h}$} \\
$Q_{i}^{1}$ & $5000 \mathrm{~kg}$ \\
$Q_{r}^{2}$ & $1500 \mathrm{~kg}$ \\
$q_{w \in W_{0}}^{1}$ & {$[20-40] \mathrm{kg}$} \\
$q_{w \in W_{1}}^{1}$ & {$[50-100] \mathrm{kg}$} \\
$q_{w \in W_{2}}^{1}$ & {$[100-200] \mathrm{kg}$} \\
\hline
\end{tabular}

TABle 4: Comparison between CPLEX solver and PSO algorithm on small-scale instances.

\begin{tabular}{|c|c|c|c|c|c|c|}
\hline \multirow{2}{*}{$\begin{array}{l}\text { Instance } \\
\text { Group }\end{array}$} & \multirow[b]{2}{*}{ ID } & \multicolumn{2}{|c|}{ CPLEX } & \multicolumn{2}{|l|}{ PSO } & \multirow[b]{2}{*}{$\mathrm{Gap}_{1}$} \\
\hline & & $F_{\text {CPLEX }}$ & $t_{\text {CPLEX }}$ & $F_{\text {PSO }}$ & $t_{\mathrm{PSO}}$ & \\
\hline \multirow{5}{*}{ ISG1 } & 1 & 11 & 1.77 & 11 & 8.10 & $0.00 \%$ \\
\hline & 2 & 11 & 1.49 & 11 & 8.22 & $0.00 \%$ \\
\hline & 3 & 11 & 0.80 & 11 & 7.83 & $0.00 \%$ \\
\hline & 4 & 11 & 1.58 & 11 & 8.31 & $0.00 \%$ \\
\hline & 5 & 12 & 1.97 & 12 & 7.60 & $0.00 \%$ \\
\hline \multirow{5}{*}{ ISG2 } & 1 & 13 & 5.17 & 13 & 15.2 & $0.00 \%$ \\
\hline & 2 & 13 & 242.43 & 13 & 14.45 & $0.00 \%$ \\
\hline & 3 & 13 & 132.15 & 13 & 14.32 & $0.00 \%$ \\
\hline & 4 & 13 & 354.97 & 13 & 14.30 & $0.00 \%$ \\
\hline & 5 & 13 & 348.38 & 13 & 13.19 & $0.00 \%$ \\
\hline \multirow{5}{*}{ ISG3 } & 1 & - & $>7200$ & 17 & 20.83 & - \\
\hline & 2 & - & $>7200$ & 17 & 21.38 & - \\
\hline & 3 & - & $>7200$ & 17 & 22.41 & - \\
\hline & 4 & - & $>7200$ & 17 & 20.79 & - \\
\hline & 5 & - & $>7200$ & 17 & 37.29 & - \\
\hline Average & - & - & - & - & 15.61 & - \\
\hline
\end{tabular}

Notes: (1) $F_{\text {CPLEX }}$ represents the optimal solution obtained by CPLEX. $F_{\text {PSO }}$ denotes the global best solution obtained by PSO algorithm. (2) $t_{\text {CPLEX }}$ and $t_{\mathrm{PSO}}$ are the computation time of CPLEX and PSO algorithm in seconds, respectively. (3) $\mathrm{Gap}_{1}=\left(\left(F_{\mathrm{PSO}}-F_{\mathrm{CPLEX}}\right) / F_{\mathrm{CPLEX}}\right)$.

Step 1. The medical waste of the clinic is collected by the nearest grade 1 hospital without exceeding its capacity. If the waste collected by a hospital has reached the capacity, then find the second nearest hospital for collecting the clinic's waste.

Step 2. The total tasks for each vehicle are proportionally distributed based on its load capacity and time windows.

Step 3. Based on the "Nearest Service" principle, each vehicle serves the closest tasks.

Table 6 illustrates the comparisons between the proposed solution methods and the rules. As can be seen, the PSO algorithm can obtain a good solution in solving the largescale problem instances, with the average computing time of 1120.28 seconds. More importantly, it is seen that the PSO algorithm outperforms the rules for all instances from ISG7
TABLE 5: Comparison between CPLEX solver and PSO algorithm on medium-scale instances.

\begin{tabular}{|c|c|c|c|c|c|c|}
\hline \multicolumn{2}{|l|}{ Instance } & \multicolumn{2}{|c|}{ CPLEX } & \multicolumn{3}{|c|}{ PSO } \\
\hline Group & ID & $F_{\text {CPLEX }}$ & $t_{\text {CPLEX }}$ & $F_{\text {PSO }}$ & $t_{\mathrm{PSO}}$ & $\mathrm{Gap}_{1}$ \\
\hline \multirow{5}{*}{ ISG4 } & 1 & 29 & $>7200$ & 28 & 51.62 & $-3.45 \%$ \\
\hline & 2 & 29 & $>7200$ & 29 & 51.64 & $0.00 \%$ \\
\hline & 3 & 28 & $>7200$ & 27 & 56.50 & $-3.57 \%$ \\
\hline & 4 & 27 & $>7200$ & 27 & 52.08 & $0.00 \%$ \\
\hline & 5 & 29 & $>7200$ & 28 & 51.93 & $-3.45 \%$ \\
\hline \multirow{5}{*}{ ISG5 } & 1 & 45 & $>7200$ & 44 & 132.59 & $-2.22 \%$ \\
\hline & 2 & 48 & $>7200$ & 47 & 129.94 & $-2.08 \%$ \\
\hline & 3 & 49 & $>7200$ & 49 & 130.73 & $0.00 \%$ \\
\hline & 4 & 48 & $>7200$ & 46 & 132.41 & $-4.17 \%$ \\
\hline & 5 & 51 & $>7200$ & 49 & 130.98 & $-3.92 \%$ \\
\hline \multirow{5}{*}{ ISG6 } & 1 & 62 & $>7200$ & 60 & 212.95 & $-3.23 \%$ \\
\hline & 2 & 69 & $>7200$ & 68 & 213.59 & $-1.45 \%$ \\
\hline & 3 & 68 & $>7200$ & 65 & 211.06 & $-4.41 \%$ \\
\hline & 4 & 66 & $>7200$ & 66 & 213.97 & $0.00 \%$ \\
\hline & 5 & 64 & $>7200$ & 62 & 215.46 & $-3.13 \%$ \\
\hline Average & - & - & - & - & 132.50 & $-2.34 \%$ \\
\hline
\end{tabular}

to ISG9 in terms of solution quality. The average gap between the two solutions is $-5.98 \%$; that is, the PSO algorithm can achieve further optimisation of the solution of rules in solving large-scale problems.

6.3. Sensitivity Analysis. The type of vehicle (e.g., capacity) has a significant influence on the optimisation of the transportation network. Therefore, we conduct a sensitivity analysis on the capacity of the vehicle to assess its effect on the objective value. Case 1, Case 2, Case 3, and Case 4 employ single type of vehicles of $500 \mathrm{~kg}, 1000 \mathrm{~kg}, 1500 \mathrm{~kg}$, and $2000 \mathrm{~kg}$, respectively, which are the four types of special transport vehicles for medical waste in practice. Case 5 represents a multivehicle fleet, which applies all types of vehicles employed. The proportions of each type of vehicles employed in Case 5 for different scales of instances are $1: 1: 1$ : $1,1: 1: 2: 1,1: 3: 3: 1$, respectively. ISG2, ISG4, and ISG7 are employed to represent the small-scale, medium-scale, and large-scale problem instances, respectively.

Figure 3 indicates that the objective value is sensitive to the capacity of the vehicle, especially for large-scale instances. In particular, the total distance shows a downward trend as the increasing capacity of single vehicle (e.g., Case 1-Case 4). There is a remarkable drop in Case 2, which means it is economical to allocate the type of $1000 \mathrm{~kg}$ vehicle for the third-party recycling company. It is worthwhile to mention that the performance of Case 5 reaches the better solution among all scale problems. Multiple vehicles can greatly improve the transportation efficiency, and the operator may allocate various types of vehicles to serve different scale tasks. 
TABLE 6: Comparison between the rules and the PSO algorithm on large-scale instances.

\begin{tabular}{|c|c|c|c|c|c|}
\hline \multicolumn{2}{|c|}{ Instance } & \multirow{2}{*}{$\begin{array}{l}\text { Rules } \\
F_{\text {Rules }}\end{array}$} & \multicolumn{3}{|c|}{ PSO } \\
\hline Group & ID & & $F_{\mathrm{PSO}}$ & $t_{\mathrm{PSO}}$ & $\mathrm{Gap}_{2}$ \\
\hline \multirow{5}{*}{ ISG7 } & 1 & 100 & 95 & 899.48 & $-5.00 \%$ \\
\hline & 2 & 99 & 91 & 864.21 & $-8.08 \%$ \\
\hline & 3 & 96 & 89 & 872.12 & $-7.29 \%$ \\
\hline & 4 & 96 & 88 & 864.62 & $-8.33 \%$ \\
\hline & 5 & 98 & 93 & 858.85 & $-5.10 \%$ \\
\hline \multirow{5}{*}{ ISG8 } & 1 & 102 & 100 & 1107.1 & $-1.96 \%$ \\
\hline & 2 & 108 & 102 & 1111.35 & $-5.56 \%$ \\
\hline & 3 & 106 & 98 & 1104.23 & $-7.55 \%$ \\
\hline & 4 & 105 & 98 & 1143.90 & $-6.67 \%$ \\
\hline & 5 & 105 & 101 & 1116.67 & $-3.81 \%$ \\
\hline \multirow{5}{*}{ ISG9 } & 1 & 125 & 117 & 1361.83 & $-6.40 \%$ \\
\hline & 2 & 119 & 114 & 1372.91 & $-4.20 \%$ \\
\hline & 3 & 123 & 118 & 1382.82 & $-4.07 \%$ \\
\hline & 4 & 131 & 120 & 1370.40 & $-8.40 \%$ \\
\hline & 5 & 124 & 115 & 1373.64 & $-7.26 \%$ \\
\hline Average & - & - & - & 1120.28 & $-5.98 \%$ \\
\hline
\end{tabular}

Notes: (1) $F_{\text {Rules }}$ represents the feasible solution obtained by rules. $F_{\text {PSO }}$ denotes the global best solution obtained by PSO algorithm. (2) $t_{\mathrm{PSO}}$ is the computation time of PSO algorithm in seconds. (3) $\mathrm{Gap}_{2}=\left(\left(F_{\mathrm{PSO}}-F_{\text {Rules }}\right) / F_{\text {Rules }}\right)$.

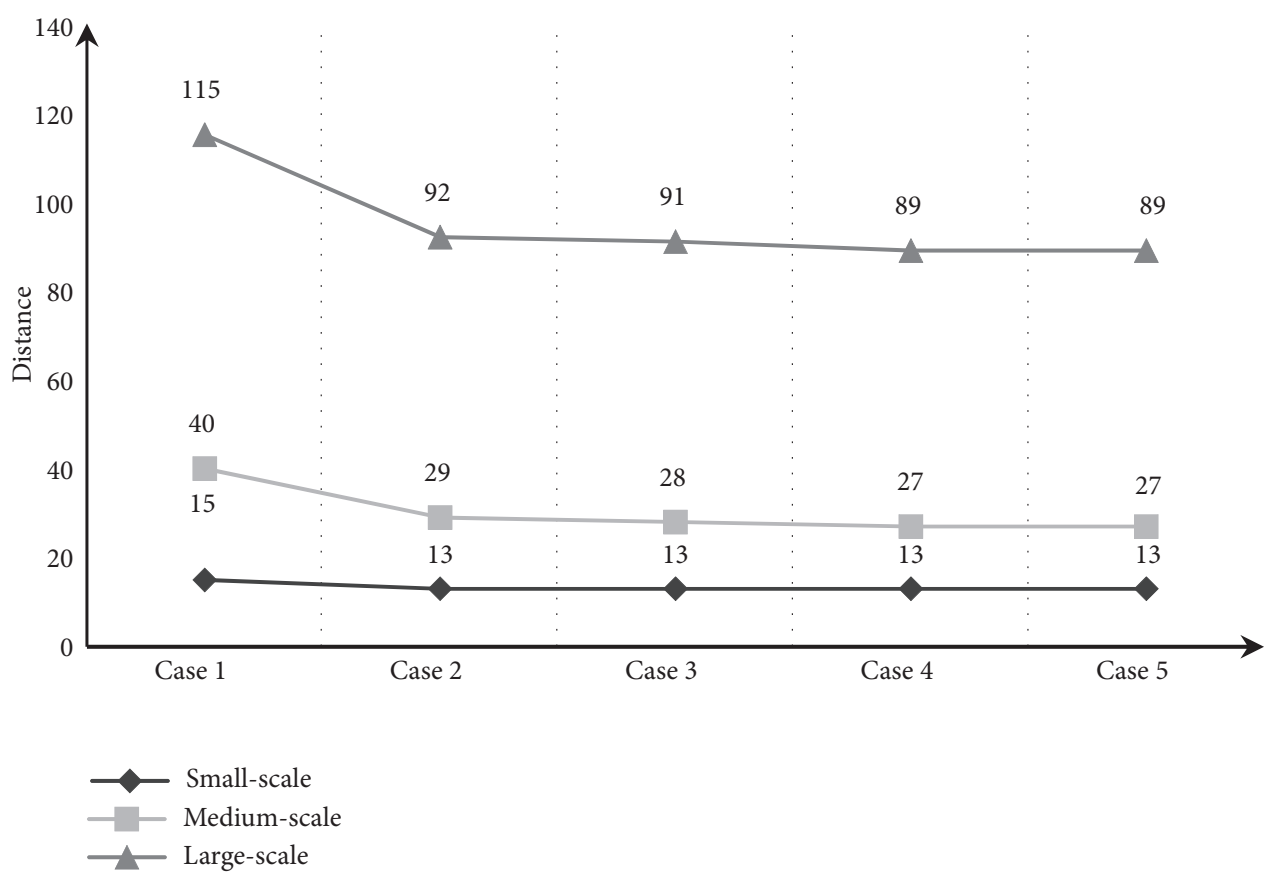

Figure 3: Sensitivity analysis about types of vehicles.

\section{Conclusion}

This paper studies an integrated optimisation problem of urban medical waste recycling network considering differentiated collection strategies with time windows. In addition, since the medical waste recycling operates according to a two-day pattern, the periodicity of the plan is also embedded in the model. To tackle the optimisation, a compact mixed-integer linear programming model is proposed, which aims to minimise the total distance of the recycling network. Then we develop a PSO solution approach to solve the problem. Based on some realistic instances, extensive numerical experiments are conducted and some managerial implications can be recommended for the third-party recycling company. The major contributions of this study are summarised in the following aspects: 
(1) This paper extends the traditional medical waste recycling network problem, which takes the periodicity of medical waste collection and differentiated medical waste collection strategies for medical facilities of various grades into account. Moreover, the affiliated relationships between clinics and grade 1 hospitals are integrated into the whole model.

(2) For solving the proposed integrated problem, this study develops a PSO solution approach, which is validated for both small-scale and large-scale instances. The PSO algorithm can obtain a good solution in solving large-scale problem instances within a reasonable time, which means the algorithm can provide a decision support tool for the operator.

(3) Based on extensive numerical experiments and sensitivity analysis, we can see that multiple vehicles can greatly improve the transportation efficiency in the recycling network. The third-party recycling company's operator may allocate various types of vehicles to serve different scale tasks.

However, there are some limitations for this study. First, the benefit of multivehicle fleet is proved by the sensitivity analysis. Multivehicle transportation can be further explored by investigating the optimal proportion of different vehicle types in a fleet. Second, although the proposed PSO algorithm can obtain solutions of high quality in reasonable time, the computation of large-scale instances still takes a longer time than expected. To improve the computation efficiency in large-scale instances, some acceleration strategies could be addressed to the algorithm. We will focus on these limitations which form the research directions for future studies.

\section{Data Availability}

No data were used to support this study.

\section{Conflicts of Interest}

The authors declare that they have no conflicts of interest.

\section{Acknowledgments}

This research was supported by the National Key R\&D Program of China (Grant no. 2018YFE0102700).

\section{References}

[1] M. Taslimi, R. Batta, and C. Kwon, "Medical waste collection considering transportation and storage risk," Computers \& Operations Research, vol. 120, p. 104966, 2020.

[2] B. A. Khan, L. Cheng, A. A. Khan, and H. Ahmed, "Healthcare waste management in Asian developing countries: a mini review," Waste Management \& Research, vol. 37, no. 9, pp. 863-875, 2019.

[3] H. Rachih, F. Z. Mhada, and R. Chiheb, "Meta-heuristics for reverse logistics: a literature review and perspectives," Computers \& Industrial Engineering, vol. 127, pp. 45-62, 2019.
[4] P. C. Nolz, N. Absi, and D. Feillet, "A stochastic inventory routing problem for infectious medical waste collection," Networks, vol. 63, no. 1, pp. 82-95, 2014.

[5] G. Mantzaras and E. A. Voudrias, "An optimization model for collection, haul, transfer, treatment and disposal of infectious medical waste: application to a Greek region," Waste Management, vol. 69, pp. 518-534, 2017.

[6] E. B. Tirkolaee, P. Abbasian, and G.-W. Weber, "Sustainable fuzzy multi-trip location-routing problem for medical waste management during the COVID-19 outbreak," Science of the Total Environment, vol. 756, p. 143607, 2021.

[7] H. Yu, X. Sun, W. D. Solvang, and X. Zhao, "Reverse logistics network design for effective management of medical waste in epidemic outbreaks: insights from the coronavirus disease 2019 (COVID-19) outbreak in Wuhan (China)," International Journal of Environmental Research and Public Health, vol. 17, no. 5, p. 1770, 2020.

[8] S. Kargar, M. M. Paydar, and A. S. Safaei, "A reverse supply chain for medical waste: a case study in Babol healthcare sector," Waste Management, vol. 113, pp. 197-209, 2020.

[9] Y. Shi, Y. J. Zhou, W. H. YE, and Q. Q. Zhao, "A relative robust optimization for a vehicle routing problem with time-window and synchronized visits considering greenhouse gas emissions," Journal of Cleaner Production, vol. 275, p. 124112, 2020.

[10] R. EL-Hajj, R. N. Guibadj, A. Moukrim, and M. Serairi, “A PSO based algorithm with an efficient optimal split procedure for the multiperiod vehicle routing problem with profit," Annals of Operations Research, vol. 291, no. 1-2, pp. 281-316, 2020.

[11] L. D. C. Martins, P. Hirsch, and A. A. Juan, "Agile optimization of a two-echelon vehicle routing problem with pickup and delivery," International Transactions in Operational Research, vol. 28, no. 5, pp. 201-221, 2021.

[12] B. O. Ovstebo, L. M. Hvattum, and K. Fagerholt, "Routing and scheduling of RoRo ships with stowage constraints," Transportation Research Part C-Emerging Technologies, vol. 19, no. 6, pp. 1225-1242, 2011.

[13] L. Zhen, "Modeling of yard congestion and optimization of yard template in container ports," Transportation Research Part B: Methodological, vol. 90, pp. 83-104, 2016.

[14] L. Zhen, "Tactical berth allocation under uncertainty," European Journal of Operational Research, vol. 247, no. 3, pp. 928-944, 2015.

[15] L. Zhen, E. P. Chew, and L. H. Lee, "An integrated model for berth template and yard template planning in transshipment hubs," Transportation Science, vol. 45, no. 4, pp. 483-504, 2011.

[16] Z.-G. He, Q. Li, and J. Fang, "The solutions and recommendations for logistics problems in the collection of medical waste in China," Procedia Environmental Sciences, vol. 31, pp. 447-456, 2016.

[17] J. Kennedy and R. Eberhart, "Particle swarm optimization," in Proceedings of ICNN'95-International Conference on Neural Networks, pp. 1942-1948, Perth, Australia, November, 1995.

[18] N. Norouzi, M. Sadegh-Amalnick, and R. Tavakkoli-Moghaddam, "Modified particle swarm optimization in a time-dependent vehicle routing problem: minimizing fuel consumption," Optimization Letters, vol. 11, no. 1, pp. 121-134, 2017.

[19] B. Yao, B. Yu, P. Hu, J. Gao, and M. Zhang, "An improved particle swarm optimization for carton heterogeneous vehicle routing problem with a collection depot," Annals of Operations Research, vol. 242, no. 2, pp. 303-320, 2016.

[20] L. Zhen, Z. Xu, K. Wang, and Y. Ding, "Multi-period yard template planning in container terminals," Transportation Research Part B: Methodological, vol. 93, pp. 700-719, 2016. 